\title{
Can Combined Screening of Ultrasound and Elastography Improve Breast Cancer Identification Compared with MRI in Women with Dense Breasts-a Multicenter Prospective Study
}

Lu-Ying Gao ${ }^{1}$, Yang Gu${ }^{1}$, Wen $\mathrm{Xu}^{1}$, Jia-Wei Tian², Li-Xue Yin ${ }^{3}$, Hai-Tao Ran ${ }^{4}$, Wei-Dong Ren ${ }^{5}$, Yu-Ming $\mathrm{Mu}^{6}$, Jie-Ying Zhang7, Cai Chang8, Jian-Jun Yuan' ${ }^{9}$, Chun-Song Kang10, You-Bin Deng11, Hui Wang12, Xiao-Yan Xie'3, Bao-Ming Luo'14, Sheng-Lan Guo ${ }^{15}$, Qi Zhou'16, En-Sheng Xue17, Wei-Wei Zhan ${ }^{18}$, Tong Jiao $^{19}$, Qing Zhou' ${ }^{20}$, Jie Li21, Ping Zhou ${ }^{22}$, Pin-Tong Huang ${ }^{23}$, Hong-Yuan Xue ${ }^{24}$, Chun-Quan Zhang25, Man $\mathrm{Chen}^{26}$, Xiang-Xiang Jing 27 , Ying $\mathrm{Gu}^{28}$, Jian-Feng Guo ${ }^{29}$, Hong-Yu Ding ${ }^{30}$, Jin-Feng $\mathrm{Xu}^{31}$, Wu Chen ${ }^{32}$, Li $\mathrm{Liu}^{33}$, Yu-Hong Zhang ${ }^{34}$, Hong-Qiao Wang ${ }^{35}$, Zhong-Ping Mu ${ }^{36}$, Jian-Chu Li1 ${ }^{1}$, Hong-Yan Wang1,,$\bowtie$, , Yu-Xin Jiang1, 1, ,

1. Department of Ultrasound, Peking Union Medical College Hospital, Chinese Academy of Medical Sciences and Peking Union Medical College, Beijing 100730, China

2. Department of Ultrasound, the Second Affiliated Hospital of Harbin Medical University, Harbin 150086, China

3. Cardiovascular Ultrasound and Non-invasive Cardiology Department, Key Laboratory of Ultrasound in Cardiac Electrophysiology and Bio-mechanics of Sichuan Province, Sichuan Academy of Medical Sciences \& Sichuan Provincial People's Hospital, Chengdu 610072, China

4. Department of Ultrasound, the Second Affiliated Hospital of Chongqing Medical University, Chongqing 400010, China; Chongqing Key Laboratory of Ultrasound Molecular Imaging, Chongqing 400010, China

5. Department of Ultrasound, Shengjing Hospital of China Medical University, Shenyang 110004, China

6. Department of Ultrasonography, the First Affiliated Hospital of Xinjiang Medical University, Urumqi 830054, China

7. Department of Radiology, Cancer Institute \&Hospital, Chinese Academy of Medical Sciences, Beijing 100730, China

8. Department of Medical Ultrasound, Fudan University Shanghai Cancer Center \& Department of Oncology, Shanghai Medical College, Fudan University, Shanghai 200032, China

9. Department of Ultrasonography, Henan Provincial People's Hospital, Zhengzhou 450003, China

10. Department of Ultrasound, Shanxi Academy of Medical Science, Dayi Hospital of Shanxi Medical University, Taiyuan 030032, China

11. Department of Medical Ultrasound, Tongji Hospital, Tongji Medical College of Huazhong University of Science and Technology, Wuhan 430030, China

12. Department of Ultrasound, China-Japan Union Hospital of Jilin University, Changchun 130033, China

13. Department of Medical Ultrasonics, Division of Interventional Ultrasound, Institute of Diagnostic and Interventional Ultrasound, the First Affiliated Hospital of Sun Yat-sen University, Guangzhou 510080, China

14. Department of Ultrasound, the Sun Yat-sen Memorial Hospital, Sun Yat-sen University, Guangzhou 510120, China

15. Department of Ultrasonography, First Affiliated Hospital of Guangxi Medical University, Nanning 530021, China

16. Department of Medical Ultrasound, the Second Affiliated Hospital, School of Medicine, Xi'an Jiaotong University, Xi'an 710004, China

17. Department of Ultrasound, Union Hospital of Fujian Medical University, Fujian Institute of Ultrasound Medicine, Fuzhou 350001, China

18. Department of Ultrasound, Ruijin Hospital, Shanghai Jiaotong University, School of Medicine, Shanghai 200025, China

19. Department of Ultrasound, Tianjin Union Medicine Hospital, Tianjin 300121, China

20. Department of Ultrasonography, Renmin Hospital of Wuhan University, Wuhan 430060, China

21. Department of Ultrasound, Qilu Hospital, Shandong University, Jinan 250012, China

22. Department of Ultrasound, the Third Xiangya Hospital of Central South University, Changsha 410013, China

23. Department of Ultrasound, the Second Affiliated Hospital of Zhejiang University, Hangzhou 310000, China

24. Department of Ultrasound, Hebei General Hospital, Shijiazhuang 050000, China

25. Department of Ultrasound, the Second Affiliated Hospital of Nanchang University, Nanchang 330006, China

26. Department of Ultrasound Medicine, Tongren Hospital, Shanghai Jiao Tong University School of Medicine, Shanghai 200336, China

27. Department of Ultrasound, Hainan Provincial People's Hospital, Haikou 570311, China

28. Department of Ultrasonography, the Affiliated Hospital of Guizhou Medical University, Guiyang 550004, China

29. Department of Ultrasound, the Affiliated Suzhou Hospital of Nanjing Medical University, Suzhou Municipal Hospital, Suzhou 215001, China

30. Department of Ultrasonography, Qianfoshan Hospital of Shandong University, Jinan 250014, China

31. Department of Ultrasound, Shenzhen People's Hospital, the Second Clinical Medical College of Jinan University, Shenzhen 518020, China

32. Department of Ultrasound, the First Hospital of Shanxi Medical University, Taiyuan 030001, China

33. Department of Ultrasound, Peking University Shenzhen Hospital, Shenzhen 518036, China

34. Department of Ultrasound, the Second Hospital of Dalian Medical University, Dalian 116027, China

35. Department of Ultrasound, the Affiliated Hospital of Qingdao University, Qingdao 266003, China

36. Department of Ultrasound, Maternal and Child Health Care Hospital Affiliated to Medical University of Anhui, Anhui Province Maternal and Child Health Hospital, Hefei 230001, China

*These authors contributed equally to this work.

$\triangle$ Corresponding author: Hong-Yan Wang; Yu-Xin Jiang, Department of Ultrasound, Peking Union Medical College Hospital, Chinese Academy of Medical Sciences and Peking Union Medical College, No.1 Shuai Fu Yuan, Dong Cheng District, Beijing 100730, China. Email address: whychina@126.com (Hong-Yan Wang ), jiangyuxinxh@163.com (Yu-Xin Jiang); Tel: +86106 915 5494; +86106915 5491; Fax: +861069155494; +861069155402 
(C) The author(s). This is an open access article distributed under the terms of the Creative Commons Attribution License (https://creativecommons.org/licenses/by/4.0/). See http://ivyspring.com/terms for full terms and conditions.

Received: 2019.12.23; Accepted: 2020.03.27; Published: 2020.04.06

\begin{abstract}
Objectives: To assess the performance of elastography (ES) and ultrasound (US) in predicting the malignancy of breast lesions and to compare their combined diagnostic value with that of magnetic resonance imaging (MRI).

Materials and Methods: The study prospectively enrolled 242 female patients with dense breasts treated in 35 heath care facilities in China between November 2018 and October 2019. Based on conventional US and elastography, radiologists classified the degree of suspicion of breast lesions according to the US Breast Imaging Reporting and Data System (BI-RADS) criteria. The diagnostic value was compared between US BI-RADS and MRI BI-RADS, with pathological results used as the reference standard.

Results: The results demonstrated that irregular tumor shape, a nonparallel growth orientation, indistinct margins, angular contours, microcalcifications, color Doppler flow and ES score on US imaging were significantly related to breast cancer in dense breasts $(P=0.001 ; P=0.001 ; P=0.008$; $\mathrm{P}<0.001 ; \mathrm{P}=0.019 ; \mathrm{P}=0.008 ; \mathrm{P}=0.002$, respectively). The sensitivity, specificity, $\mathrm{PPV}, \mathrm{NPV}$, accuracy and AUC of US BI-RADS category were $94.7 \%, 90.7 \%, 95.8 \%, 88.0 \%, 93.4 \%$ and $0.93(95 \% \mathrm{Cl}$, 0.88-0.97), respectively, while those of MRI BI-RADS category were $98.2 \%, 57.5 \%, 84.3 \%, 83.3 \%$, $86.0 \%$ and $0.78(95 \% \mathrm{Cl}, 0.71-0.85)$, respectively. MRI BI-RADS showed a significantly higher sensitivity than US BI-RADS ( $98.2 \%$ vs $94.7 \%, P=0.043)$, whereas US BI-RADS showed significantly higher specificity $(90.7 \%$ vs $57.5 \%, P<0.001)$. US BI-RADS showed better diagnostic efficiency in differentiating nodules in dense breasts than MRI BI-RADS (AUC 0.93 vs $0.78, P<0.001$ ).

Conclusion: By combining the use of ES and conventional US, US BI-RADS had better diagnostic efficiency in differentiating nodules in dense breasts than MRI. For the diagnosis of malignant tumors in patients with dense breasts, MRI and US BI-RADS can be used as supplemental diagnostic tools to detect lesions, with US BI-RADS considered the preferred adjunctive resource.
\end{abstract}

Key words: Breast cancer; Dense breast; Breast Imaging Reporting and Data System (BI-RADS); Elastography; Magnetic resonance imaging (MRI); Ultrasound (US)

\section{Introduction}

Mammography is a standard screening test that has been proven to reduce breast cancer-related mortality $[1,2]$. However, dense glandular structures reduce the sensitivity of mammography, causing delayed diagnosis and worse outcomes [3]. Ultrasound and magnetic resonance imaging (MRI) as adjuncts to mammography can aid in assessing breast lesions in dense breasts. US is a commonly used modality for detecting early breast cancers in dense breasts $[4,5]$. However, the sonographic appearance of benign and malignant nodules overlaps to some extent, causing overtreatment biopsies that are a major limitation of US. US elastography (ES) is a new technique that improves the diagnostic value of US $[6,7]$. ES has been incorporated into the fifth edition of the Breast Imaging Report and Data System (BI-RADS) of the American College of Radiology (ACR), which suggests the risk stratification of breast lesions based on suspicious conventional US features and elastography [8].

MRI has been considered the most sensitive screening modality for breast cancer. However, its average specificity is relatively low and varies according to the tumor indications [9]. A previous study performed in Korea showed that the addition of elastography and color Doppler US to B-mode US can increase the PPV of screening US in women with dense breasts [10]. However, they did not include MRI information and failed to compare the diagnostic value of US and MRI for lesions in dense breasts. The objective of this study was to explore the potential of combining US and US elastography and compare the diagnostic value of US and MRI for predicting breast cancer in dense breasts.

\section{Materials and Methods}

\section{Patients}

This was a multicenter study conducted at regional medical centers in China, including 35 hospitals from 23 different provinces. All hospitals completed real-name registration on the website (www.nuqcc.cn) and a data survey after approval. The study prospectively enrolled 3292 patients with 
breast lesions who underwent biopsy or surgery at the 35 hospitals between November 2018 and October 2019. The following inclusion criteria were applied: (1) female patients older than 18 years of age; (2) patients assessed as having dense breast by mammography; and (3) patients for whom conventional US, elastography and MRI screening were performed. Patients who received treatment before surgery were excluded. A total of 242 patients were finally included [Figure 1]. All of these patients underwent breast ultrasound examination prior to core needle biopsy or surgical pathology. The final pathologic results were considered the diagnostic gold standard. The clinical features of the patients were recorded.

\section{Breast examination and prospective evaluation}

All US examinations were performed with Resona7 or 8 devices (Mindray Medical, Shenzhen, China) equipped with $5-14 \mathrm{MHz}$ linear-array transducers. US images were prospectively evaluated by 35 radiologists who were experienced in breast US and were blinded to the patient clinical data. First, conventional US images of the lesions were obtained, including B-mode US and color Doppler images. The tumor size, shape, echogenicity, growth orientation, margin, and contour, the presence of architectural distortion, the presence of duct ectasia, acoustic shadowing and microcalcifications were evaluated by B-mode US. Vascularity was classified into 4 patterns (no flow, minimal, moderate, or marked) by color Doppler flow [11]. After conventional US, elastography images were generated by the same radiologists. Each lesion was assigned an elasticity score according to a 5-point scoring system [12]. Based on B-mode US, color Doppler and elastography, the radiologists classified the degree of suspicion of breast lesions according to the BI-RADS criteria [8]. MRI images were acquired on different scanners at the 35 referring region medical centers. All examination protocols included a T2-weighted and/or STIR sequence as well as T1-weighted contrast-enhanced dynamic images before and after single-dose Gd-based contrast media injection at 1.5 or $3 \mathrm{~T}$, all in line with EUSOBI and EUSOMA recommendations $[13,14]$. Based on the MRI, the radiologists classified the degree of suspicion of breast lesions according to the BI-RADS criteria [8]. All radiologists completed real-name registration on the website (www.nuqcc.cn), and all the patients' images were uploaded. All the data and images from the website were separately reviewed by three experienced radiologists in our hospitals. In cases involving a discrepancy a consensus was reached after discussion.

\section{Statistical analysis}

Quantitative data are presented as the means \pm standard deviations (SDs). Qualitative data are presented as frequencies. To assess the correlations between features and axillary lymph node metastasis, the $\chi^{2}$-test was used. The sensitivity, specificity, positive predictive value (PPV), negative predictive value (NPV) and accuracy were calculated through a comparison with the pathological findings. Receiver operating characteristic (ROC) curve analysis was performed. A $\mathrm{P}$ value $<0.05$ was considered statistically significant. Statistical analyses were performed with SPSS software (Version 19.0, SPSS Chicago, IL, USA) and MedCalc 11.4.2.0 software (MedCalc Software, Ostend, Belgium).

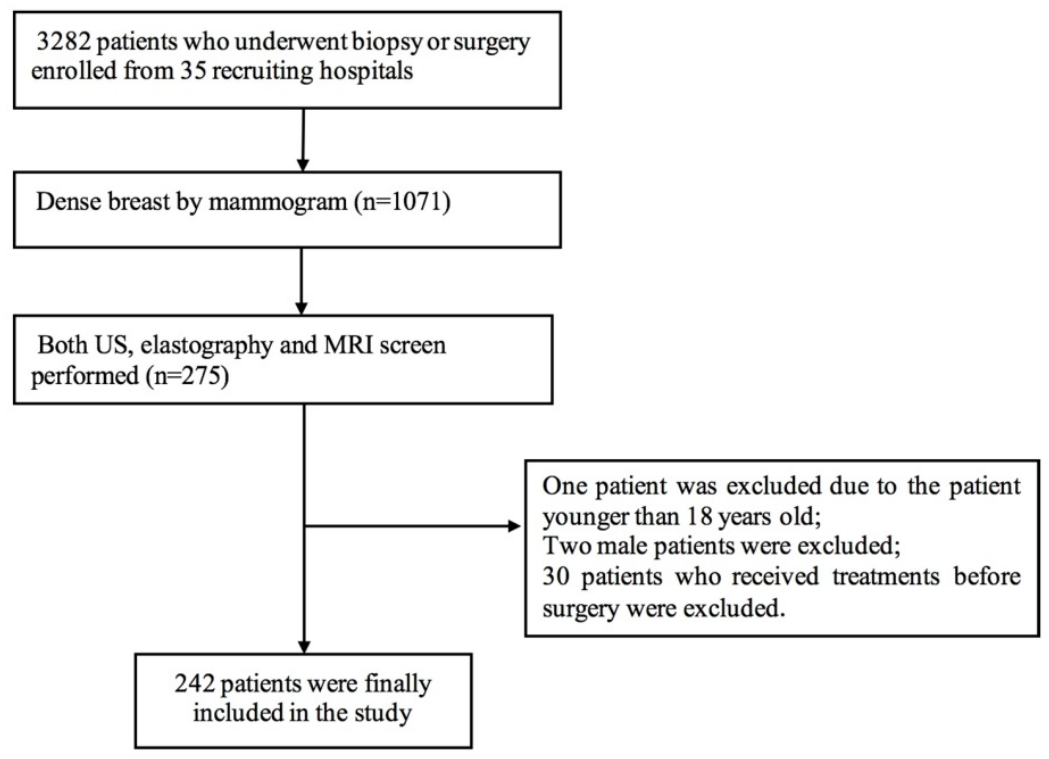


Table 1. Clinical and imaging characteristics of benign and malignant breast lesions

\begin{tabular}{|c|c|c|c|c|}
\hline & $\begin{array}{l}\text { Benign, } \\
\mathrm{n}(\%)\end{array}$ & $\begin{array}{l}\text { Malignant, } \\
\mathrm{n}(\%)\end{array}$ & Total & $\mathrm{P}$ \\
\hline Age & $44.5 \pm 9.4$ & $49.3 \pm 10.6$ & & 0.001 \\
\hline Age & & & & $<0.001$ \\
\hline$<50$ & $55(39.0)$ & $86(61.0)$ & 141 & \\
\hline$\geq 50$ & 18(17.8) & $83(82.2)$ & 101 & \\
\hline First-degree relatives with breast cancer & & & & 0.70 \\
\hline 0 & $73(30.3)$ & $168(69.7)$ & 241 & \\
\hline 1 & $0(0.0)$ & $1(100.0)$ & 1 & \\
\hline Tumor size $(\mathrm{cm})$ & & & & $<0.001$ \\
\hline$<2$ & $68(57.1)$ & $51(42.9)$ & 119 & \\
\hline$\geq 2$ & 15(12.2) & $108(87.8)$ & 123 & \\
\hline Tumor shape & & & & 0.012 \\
\hline Round, oval & $73(31.7)$ & $157(68.3)$ & 230 & \\
\hline Irregular & $0(0.0)$ & $12(100.0)$ & 12 & \\
\hline Growth orientation & & & & $<0.001$ \\
\hline Parallel & $68(37.0)$ & $116(63.0)$ & 184 & \\
\hline Nonparallel & $5(8.6)$ & $53(91.4)$ & 58 & \\
\hline Margin & & & & $<0.001$ \\
\hline Circumscribed & $22(12.0)$ & $162(88.0)$ & 184 & \\
\hline Indistinct & $51(87.9)$ & $7(12.1)$ & 58 & \\
\hline Contour & & & & $<0.001$ \\
\hline Smooth, lobulated & $66(45.8)$ & $78(54.2)$ & 144 & \\
\hline Angular & $7(7.1)$ & 91(92.9) & 98 & \\
\hline Acoustic shadowing & & & & 0.51 \\
\hline Yes & $11(28.9)$ & $27(71.1)$ & 38 & \\
\hline $\mathrm{NO}$ & $62(30.4)$ & $142(69.6)$ & 204 & \\
\hline Microcalcification & & & & $<0.001$ \\
\hline Yes & $11(28.9)$ & $27(71.1)$ & 38 & \\
\hline NO & $62(30.4)$ & $142(69.6)$ & 204 & \\
\hline Echogenicity & & & & 0.15 \\
\hline Hypo-echoic & $63(28.9)$ & 155(71.1) & 218 & \\
\hline Complex & $10(41.7)$ & $14(58.3)$ & 24 & \\
\hline Presence of architectural distortion & & & & $<0.001$ \\
\hline Yes & $0(0.0)$ & $33(100.0)$ & 33 & \\
\hline No & $73(34.9)$ & $136(65.1)$ & 209 & \\
\hline Presence of duct ectasia & & & & 0.46 \\
\hline Yes & $5(26.3)$ & 14(73.7) & 19 & \\
\hline No & $68(30.5)$ & $155(69.5)$ & 223 & \\
\hline CDFI level & & & & $<0.001$ \\
\hline $0-1$ & $54(57.4)$ & $40(42.6)$ & 94 & \\
\hline $2-3$ & 19(12.8) & $129(87.2)$ & 148 & \\
\hline ES score & & & & $<0.001$ \\
\hline 1 & $5(71.4)$ & $2(28.6)$ & 7 & \\
\hline 2 & $22(75.9)$ & $7(24.1)$ & 29 & \\
\hline 3 & $36(48.6)$ & $38(51.4)$ & 74 & \\
\hline 4 & $10(11.2)$ & $79(88.8)$ & 89 & \\
\hline 5 & $0(0.0)$ & $43(100.0)$ & 43 & \\
\hline ES score & & & & $<0.001$ \\
\hline $1-3$ & $63(57.3)$ & $47(42.7)$ & 110 & \\
\hline $4-5$ & $10(7.6)$ & $122(92.4)$ & 132 & \\
\hline US BI-RADS category & & & & $<0.001$ \\
\hline 2 & $1(100.0)$ & $0(0.0)$ & 1 & \\
\hline 3 & $26(96.3)$ & 1(3.7) & 27 & \\
\hline $4 a$ & $39(83.0)$ & $8(17.0)$ & 47 & \\
\hline $4 b$ & $5(16.1)$ & $26(83.9)$ & 31 & \\
\hline $4 c$ & $2(2.6)$ & $76(97.4)$ & 78 & \\
\hline 5 & $0(0.0)$ & $58(100.0)$ & 58 & \\
\hline US BI-RADS category & & & & $<0.001$ \\
\hline $3-4 b$ & $66(88.0)$ & $9(12.0)$ & 75 & \\
\hline $4 b-5$ & $7(4.2)$ & $160(95.8)$ & 167 & \\
\hline MRI BI-RADS category & & & & $<0.001$ \\
\hline 1 & $4(80.0)$ & $1(20.0)$ & 5 & \\
\hline 2 & $8(100.0)$ & $0(0.0)$ & 8 & \\
\hline 3 & $30(93.8)$ & $2(6.3)$ & 32 & \\
\hline 4 & $31(20.8)$ & $118(79.2)$ & 149 & \\
\hline 5 & $0(0.0)$ & $48(100.0)$ & 48 & \\
\hline MRI BI-RADS category & & & & $<0.001$ \\
\hline $0-3$ & 42(93.3) & $3(6.7)$ & 45 & \\
\hline $4-5$ & $31(15.7)$ & $166(84.3)$ & 197 & \\
\hline
\end{tabular}

Abbreviations: BI-RADS: Breast Imaging-Reporting and Data System; US: ultrasound; MRI: magnetic resonance imaging; ES: elastography; CDFI: color doppler flow imaging

\section{Results}

\section{Clinical and pathological status of the patients}

The age range of the included patients was 20 to 77 years (median, 48 years). Based on pathology, 169 cases $(69.8 \%)$ had breast cancer, and $73(30.2 \%)$ did not. Among the patients with breast cancer, 99 had invasive carcinoma, 39 had intraductal carcinoma, one had mucinous carcinoma, 2 had adenocarcinoma, 3 had medullary carcinoma, 3 had a malignant phyllodes tumor, 5 had lobular carcinoma in situ, 3 had ductal carcinoma in situ, 3 had carcinoma with neuroendocrine features, 4 had micropapillary carcinoma, 2 had tubular carcinoma, 4 had papillary carcinoma and one had lymphoma. Among the patients without breast cancer, 27 had fibrocystic disease and adenosis, 26 had fibroadenoma, 10 had intraductal papilloma, 2 had inflammatory and related lesions, one had papilloma associated with fibroadenoma, a benign phyllodes tumor, 2 had a cyst, one case of fat necrosis, one case of sclerosing adenosis, one case of benign breast tissue, and one case of stromal fibrosis [Table 1].

\section{Clinical and imaging characteristics of benign and malignant breast lesions}

Comparisons of US and clinical features between malignant and benign lesions are shown in Table 1. There were significant differences in age $(\mathrm{P}<0.001)$, tumor size $(\mathrm{P}<0.001)$, tumor shape $(\mathrm{P}=0.012)$, growth orientation $(\mathrm{P}<0.001)$, margin $(\mathrm{P}<0.001)$, contour $(\mathrm{P}<0.001)$, microcalcifications $(\mathrm{P}<0.001)$, presence of architectural distortions $(\mathrm{P}<0.001)$, color Doppler flow $(\mathrm{P}<0.001)$, elastography $(\mathrm{ES})$ score $(\mathrm{P}<0.001)$ and US BI-RADS category $(\mathrm{P}<0.001)$ between the malignant and benign groups. However, echogenicity $(\mathrm{P}=0.15)$, acoustic shadowing $(\mathrm{P}=0.51)$ and the presence of duct ectasia $(P=0.46)$ were not associated with lesion malignancy [Table 1]. The overall MRI BI-RADS categories were also significantly different $(\mathrm{P}<0.001)$ between the malignant and benign groups.

\section{Diagnostic performance of US and MRI BI-RADS by category}

In terms of the US BI-RADS category, the ROC curves demonstrated that the best cut-off value was US BI-RADS $4 \mathrm{~b}$. The sensitivity, specificity, PPV, NPV, accuracy and AUC were 94.7\%, 90.7\%, 95.8\%, $88.0 \%, 93.4 \%$ and 0.93 (95\%CI, 0.88-0.97), respectively. In terms of the MRI BI-RADS category, the ROC curves demonstrated that the best cut-off value was MRI BI-RADS 4. The sensitivity, specificity, PPV, 
NPV, accuracy and AUC were 98.2\%, 57.5\%, 84.3\%, $83.3 \%, 86.0 \%$ and 0.78 (95\%CI, 0.71-0.85), respectively. When combining the optimal US BI-RADS and MRI BI-RADS categories, the sensitivity, specificity, PPV, NPV, accuracy, and AUC were 92.9\%, 91.8\%, 96.3\%, $84.8 \%, 92.6 \%$ and 0.93 (95\% CI, 0.88-0.97), respectively.

The diagnostic value of the US BI-RADS category was better than that of the MRI BI-RADS category alone (AUC 0.93 vs $0.78, \mathrm{P}<0.001$ ). The US BI-RADS category showed a significantly higher specificity than the MRI BI-RADS category $(90.7 \%$ vs $57.5 \%, \mathrm{P}<0.001$ ), whereas the MRI BI-RADS category yielded a higher sensitivity ( $98.2 \%$ vs $94.7 \%, \mathrm{P}=0.043$ ). The diagnostic value of combining the US BI-RADS with the MRI BI-RADS category is equal to the US BI-RADS stratification alone (AUC 0.93 vs 0.93, $\mathrm{P}=0.81$ ) [Table 2].

\section{Discussion}

Dense breasts can hide nodules on mammography, and a negative result on mammography does not reliably exclude the presence of breast lesions. The most widely available supplemental screening options for patients with dense breast tissue are US and MRI, but there has been a lack of information to guide the decision to utilize one or the other versus both. The results of the present study showed that US showed a higher specificity for detecting malignant breast lesions, whereas MRI yielded a higher sensitivity. Overall, US showed better diagnostic efficiency in differentiating nodules in dense breasts than MRI. The results also showed that the diagnostic value of US and MRI combined was equal to the value of US alone.

US has been applied to more than 200,000 women and is capable of increased lesion detection in addition to mammography for women with dense breasts, similar to the results of this study [15-16]. Among the conventional US characteristics of breast lesions, this study showed that irregular tumor shape, nonparallel growth orientation, indistinct margin, angular contour, microcalcification and color Doppler flow on US imaging were significantly related to breast cancer in dense breasts. However, there are several barriers to implementing screening US in practice. One of these has been the high rates of false positives from US. A recent study showed a PPV of $48 \%$ for biopsies performed based on the results of screening US in women at average risk for breast cancer with dense breasts [17].

Table 2. Diagnostic efficiency of US and MRI BI-RADS for predicting breast cancer in dense breasts

\begin{tabular}{|c|c|c|c|c|c|c|}
\hline & Sensitivity (\%) & Specificity $(\%)$ & PPV (\%) & NPV (\%) & Accuracy (\%) & AUC $(95 \% \mathrm{CI})$ \\
\hline MRI BI-RADS category & 98.2 & 57.5 & 84.3 & 83.3 & 86.0 & $0.78(0.71-0.85)$ \\
\hline US BI-RADS category & 94.7 & 90.7 & 95.8 & 88.0 & 93.4 & $0.93(0.88-0.97)$ \\
\hline MRI and US BI-RADS category & 92.9 & 91.8 & 96.3 & 84.8 & 92.6 & $0.93(0.88-0.97)$ \\
\hline
\end{tabular}

Abbreviations: BI-RADS: Breast Imaging-Reporting and Data System; US: ultrasound; MRI: magnetic resonance imaging
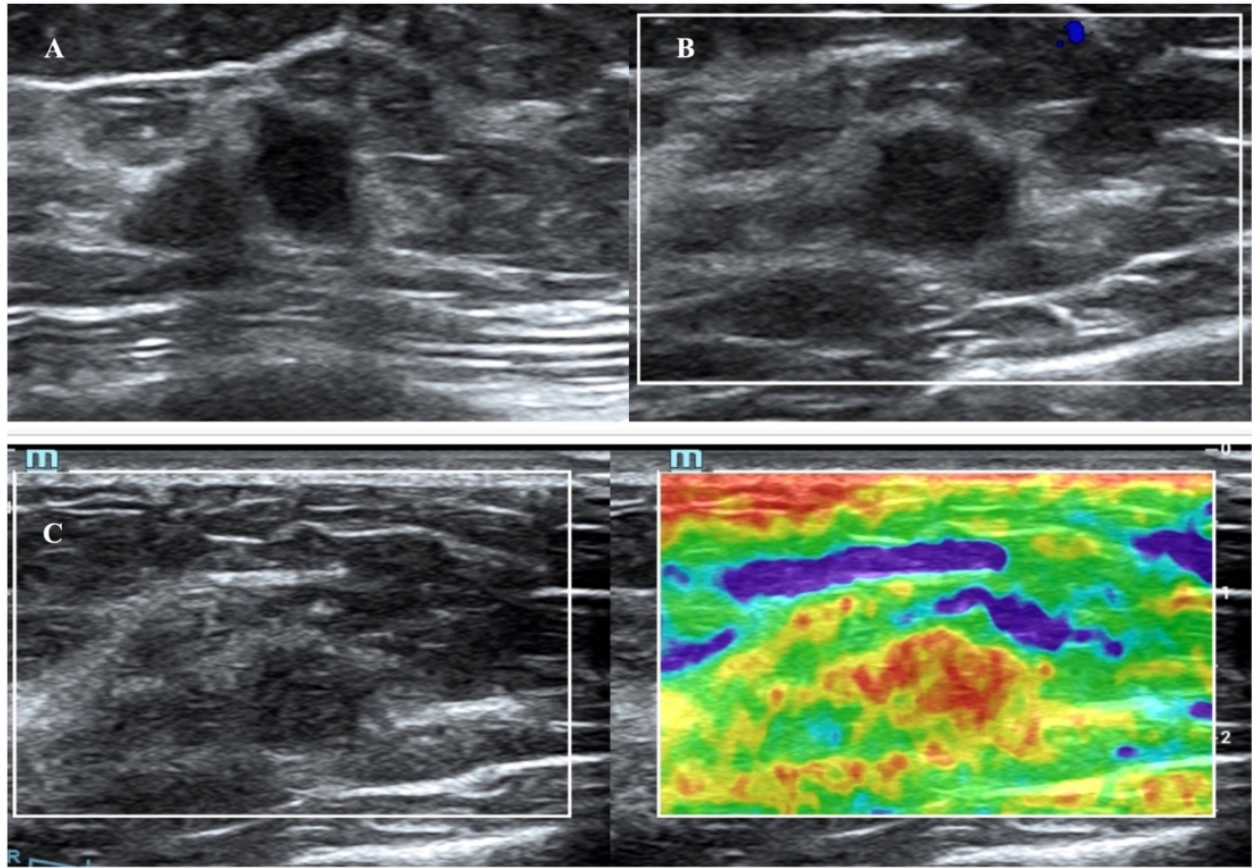

Figure 2. A breast mass from a 64-year-old woman with invasive carcinoma, which was classified as US BI-RADS category 4b and MRI BI-RADS category 3. B-mode US showed a 1.3-cm hypoechoic mass (A). The color Doppler ultrasound image of the same mass reveals no internal or peripheral blood flow signal (B). The elastographic image showed that the lesion was scored as a 5 with the 5 -point method (C). 
The revisions to the BI-RADS Fifth Edition (2013) grant ES a complementary role in the ultrasonic diagnosis of breast nodules. A 5-point scale was adopted according to the hardness of the nodules in strain elastography [18]. Previous studies have reported an increase in accuracy when combining B-mode US and ES [19-20]. A previous study reported that the addition of elastography and color Doppler US to B-mode US in dense breasts increased the AUC to 0.96 and specificity to $76.4 \%$ without loss of sensitivity [10]. In this study, similarly, the highest AUC and specificity of US were achieved when elastography was added to conventional US for diagnosing breast cancer in dense breasts. Moreover, the fifth BI-RADS category had a PPV of $95.8 \%$, and the high PPV helped reduce the number of false-positive findings without missing cancers. A relatively high PPV helps reduce unnecessary biopsies.

For high-risk women of any breast density, supplemental screening with annual MRI has been proven to reduce late-stage disease and increase metastasis-free survival [21-22]. According to a systematic review, the use of MRI for high-risk women improved the sensitivity of lesion detection by mammography from 32 to $84 \%$ [23]. Moreover, the use of supplemental MRI screening in women with extremely dense breast tissue and normal results on mammography resulted in the diagnosis of significantly fewer interval cancers than mammography alone [24-25]. MRI has been considered the most sensitive screening modality for women with dense breasts [26]. In this study, MRI also performed well in differentiating breast nodules in dense breasts, exhibiting a higher sensitivity (98.2\%) than US. Previous studies have shown that conventional ultrasound did not improve detection over MRIs and that conventional US leads to a greater number of false-positive breast cancers compared to MRI [27]. In this study, combined with ES, US showed higher specificity and had a better diagnostic efficiency in differentiating nodules in dense breasts than MRI.

There are several limitations to the study. First, the 35 hospitals are referral cancer centers instead of community hospitals, which may have resulted in an increase in the malignancy ratio of the nodules in the patient population. Second, all of the patients had pathology results. Therefore, the study included more malignant breast nodules than benign nodules, which may have led to selection bias and resulted in the underestimation of the NPV and the overestimation of the PPV. Third, potential biases may have been present because the many lesions detected during the study period were not subjected to MRI examinations, as these were performed at the discretion of the patient. Fourth, because 35 radiologists performed the MRI and US examinations, there may have been interobserver differences.

In conclusion, this study found that tumor shape, growth orientation, margin, microcalcification, color Doppler flow and ES score on US were independently associated with breast cancer in patients with dense breasts. US BI-RADS showed a higher specificity, and MRI yielded a higher sensitivity. US BI-RADS had a better diagnostic efficiency in differentiating nodules in dense breasts than MRI. For the diagnosis of malignancy in patients with dense breasts, MRI and US BI-RADS are supplemental diagnostic tools to detect lesions, with US BI-RADS being the preferred adjunctive resource.

\section{Abbreviations}

BI-RADS: Breast Imaging-Reporting and Data System; ACR: American College of Radiology; US: ultrasound; MRI: magnetic resonance imaging; PPV: positive predictive value; NPV: negative predictive value; ROC: receiver operating curve; ES: elastography; SD: standard deviation; CDFI: color doppler flow imaging.

\section{Acknowledgements}

\section{Funding}

This work is supported by the National Natural Science Foundation of China (81601517), Natural Science Foundation of Beijing (7202156), the Foundation of ihecc2018C0032-2 and Peking Union Medical College Reform in Education Project (10023201900113).

\section{Ethical approval}

This study was approved by the ethics committee of the principal investigator's hospital (Peking Union Medical College Hospital) and is registered at ClinicalTrials.gov (ChiCTR1900023916). All procedures performed in studies were in accordance with the 1964 Helsinki declaration and its later amendments or comparable ethical standards. Informed consent was obtained from all individual participants included in the study.

\section{Author Contributions}

G-LY and GY participated in the study design, performed the statistical analysis, and drafted the manuscript. L-JC conceived of the study, participated in its design. XW, Z-JY, T-JW, Y-LX, R-HT, R-WD, M-WD, CC, Y-JJ, K-CS, D-YB, WH, X-XY, L-BM, G-LS, G-SL, ZQ, X-ES, Z-WW, JT, ZQ, LJ, ZP, H-PT, X-HY, Z-CQ, CM, J-XX, GY, G-JF, D-YH, X-JF, CW, LL, $\mathrm{Z}-\mathrm{YH}, \mathrm{W}-\mathrm{HQ}$, and M-ZP carried out the selection and 
collection of samples. W-HY and J-YX participated in the study design, carried out the identification of complex performed the statistical analysis, and review the manuscript. All authors read and approved the final manuscript.

\section{Competing Interests}

The authors have declared that no competing interest exists.

\section{References}

1. Independent UK Panel on Breast Cancer Screening. The benefits and harms of breast cancer screening: an independent review. Lancet. 2012; 380: 1778-1786.

2. Tabár L, Vitak B, Chen $\mathrm{TH}$, et al. Swedish two-county trial: impact of mammographic screening on breast cancer mortality during 3 decades. Radiology. 2011; 260: 658-663.

3. Kolb TM, Lichy I, Newhouse JH. Comparison of the performance of screening mammography, physical examination, and breast US and evaluation of factors that influence them: an analysis of 27,825 patient evaluations. Radiology. 2002; 225: 165-175.

4. Shen $\mathrm{S}$, Zhou $\mathrm{Y}, \mathrm{Xu} \mathrm{Y}$, et al. A multi-centre randomised trial comparing ultrasound vs mammography for screening breast cancer in high-risk Chinese women. Br J Cancer. 2015; 112: 998-1004.

5. Health Quality Ontario. Ultrasound as an Adjunct to Mammography for Breast Cancer Screening: A Health Technology Assessment. Ont Health Technol Assess Ser. 2016; 16: 1-71.

6. Zhi H, Xiao XY, Ou B, et al. Could ultrasonic elastography help the diagnosis of small $(\leq 2 \mathrm{~cm})$ breast cancer with the usage of sonographic BI-RADS classification? Eur J Radiol. 2012; 81: 3216-21.

7. Lee JH, Kim SH, Kang BJ, et al. Role and clinical usefulness of elastography in small breast masses. Acad Radiol. 2011; 18: 74-80.

8. American College of Radiology. Breast Imaging Reporting and Data System. Breast imaging atlas. 5th edition. Reston, VA: Author 2013.

9. Peters $\mathrm{N}$, Borel $\mathrm{R}$, Zuithoff $\mathrm{N}$, et al. Meta-analysis of MR imaging in the diagnosis of breast lesions. Radiology. 2008; 246: 116-124.

10. Lee SH, Chung J, Choi HY, et al. Evaluation of Screening US-detected Breast Masses by Combined Use of Elastography and Color Doppler US with B-Mode US in Women with Dense Breasts: A Multicenter Prospective Study. Radiology. 2017; 285: 660-669.

11. Adler DD, Carson PL, Rubin JM, et al. Doppler ultrasound color flow imaging in the study of breast cancer: Preliminary findings. Ultrasound Med Biol. 1990; 16: 553-559.

12. Itoh $\mathrm{A}$, Ueno $\mathrm{E}$, Tohno $\mathrm{E}$, et al. Breast disease: Clinical application of US elastography for diagnosis. Radiology. 2006; 239: 341-350.

13. Mann R M, Kuhl C K, Kinkel K, et al. Breast MRI: guidelines from the European Society of Breast Imaging. European Radiology. 2008; 18:1307-1318.

14. Sardanelli F, Boetes C, Borisch B, et al. Magnetic resonance imaging of the breast: Recommendations from the EUSOMA working group. Eur J Cancer. 2010; 46:0-1316

15. Elizabeth H Dibble, Tisha M Singer, Nneka Jimoh, et al. Dense breast ultrasound screening after digital mammography versus after digital breast tomosynthesis. R Am J Roentgenol.2019;213:1397-1402

16. Berg WA. Current Status of Supplemental Screening in Dense Breasts. J Clin Oncol.2016;34,1840-1843

17. Tagliafico A S, Calabrese M, Mariscotti G, et al. Adjunct Screening With Tomosynthesis or Ultrasound in Women With Mammography-Negative Dense Breasts: Interim Report of a Prospective Comparative Trial. J Clin Oncol. 2016; 34: 1882-1888

18. Itoh $\mathrm{A}$, Ueno $\mathrm{E}$, Tohno $\mathrm{E}$, et al. Breast disease: Clinical application of US elastography for diagnosis. Radiology. 2006; 239: 341-350.

19. Zhi H, Xiao XY, Ou B, et al. Could ultrasonic elastography help the diagnosis of small $(\leq 2 \mathrm{~cm})$ breast cancer with the usage of sonographic BI-RADS classification? Eur J Radiol. 2012; 81: 3216-21

20. Lee JH, Kim SH, Kang BJ, et al. Role and clinical usefulness of elastography in small breast masses. Acad Radiol. 2011; 18: 74-80.

21. Saslow D, Boetes C, Burke W, et al: American Cancer Society guidelines for breast screening with MRI as an adjunct to mammography. CA Cancer J Clin. 2007; 57: 75-89

22. Warner E, Hill K, Causer $\mathrm{P}$, et al. Prospective study of breast cancer incidence in women with a BRCA1 or BRCA2 mutation under surveillance with and without magnetic resonance imaging. J Clin Oncol. 2011; 29:1664-1669

23. Warner $\mathrm{E}$, Messersmith $\mathrm{H}$, Causer $\mathrm{P}$, et al. Systematic review: using magnetic resonance imaging to screen women at high risk for breast cancer. Ann Intern Med. 2008; 148:671-679

24. Bakker MF, de Lange SV, Pijnappel RM, et al. Supplemental MRI screening for women with extremely dense breast tissue. N Engl J Med. 2019;381:2091-2102.

25. Longo DL. Detecting Breast Cancer in Women with Dense Breasts. N Engl J Med. 2019; 381:2169-2170.
26. Bu Y, Xia J, Joseph B, et al. Non-contrast MRI for breast screening: preliminary study on detectability of benign and malignant lesions in women with dense breasts. Breast Cancer Res Treat. 2019;177:629-639.

27. Berg WA, Blume JD, Cormack JB, et al. Combined screening with ultrasound and mammography vs mammography alone in women at elevated risk of breast cancer. JAMA. 2008; 299: 2151-2163. 